Asian J. Med. Biol. Res. 2020, 6 (3), 366-374; doi: 10.3329/ajmbr.v6i3.49784

\author{
Asian Journal of \\ Medical and Biological Research \\ ISSN 2411-4472 (Print) 2412-5571 (Online) \\ www.ebupress.com/journal/ajmbr
}

Review

\title{
Role of macrophages in atherosclerosis
}

\author{
Afrin Sultana Chowdhury ${ }^{*}$, Saheda Tamanna and Kumkum Kar \\ Department of Biotechnology and Genetic Engineering, Noakhali Science and Technology University, \\ Noakhali, Bangladesh
}

*Corresponding author: Afrin Sultana Chowdhury, Department of Biotechnology and Genetic Engineering, Noakhali Science and Technology University, Noakhali, Bangladesh. Phone: +8801834066794; E-mail: afrin.bge@nstu.edu.bd

Received: 27 July 2020/Accepted: 25 August 2020/ Published: 30 September 2020

\begin{abstract}
Atherosclerosis is a chronic inflammatory state, which arise from the imbalance in lipid metabolism. Over the last decade, studies have showing the association of macrophages with this maladaptive immune response. Macrophages differentiated from monocytes and populate at the growing atherosclerotic lesions. At the lesion site by accumulating lipid they actively participate in the formation of atherosclerotic plaque. These plaques are very susceptible to rupture which can lead to myocardial infarction or stroke. In future more studies are needed to classify different macrophage populations according to their phenotypic and functional characteristics to identify their roles in the pathogenesis of atherosclerosis. This review highlights several aspects of macrophages activation, diversity, recruitment, and foam cell formation in atherosclerosis.
\end{abstract}

Keywords: atherosclerosis; macrophages; chemokines

\section{Introduction}

Atherosclerosis and its associated risk factor hypercholesterolemia causes 16.7 million deaths each year worldwide (Dahlöf, 2010; Lloyd-Jones, 2010). Atherosclerosis has been considered as a slowly progressing chronic inflammatory disease of the arterial walls (Back and Hansson, 2015). The branching points and bends of arteries are particularly susceptible to the formation of atherosclerotic lesion due to the disturbance of local endothelial functions. According to present knowledge, circulating low-density lipoprotein (LDL) molecules acts as a primary source of lipid accumulation in atherosclerotic lesions by entering the vulnerable areas of the arterial vasculature (Krauss, 2010). The accumulation of lipid form plaque at the atherosclerotic lesion which gradually reduce blood flow in the arteries. This eventually causes a complete blockage of the artery (Weber $e t$ $a l ., 2008$ ) and result in insufficient supply of oxygen to the heart, or peripheral arteries.

The typical pathology of atherosclerosis is the accumulation of modified lipids, migration of inflammatory cells, formation of foam cells, and changes in the extracellular matrix (ECM) (Gronholdt et al., 1998; Shah, 2002). Over the last few years, after studying a variety of experimental animals of atherosclerosis it has become increasingly clear that inflammation and immunity are tightly associated with atherosclerosis. Knowing the basics of the inflammatory processes is essential for understanding the complex mechanisms involve in atherosclerosis progression, not only in animal models but in humans as well.

Macrophages are the inflammatory cells which are first recruited to the atherosclerotic lesion sites and play a key role at all stages of atherosclerotic lesion progression. It is widely accepted that circulating monocytederived cells that enter the atherosclerotic lesions, further differentiate into macrophages. However, some recent studies have reported that macrophages can develop from precursor cells present in adult tissues, independently of the monocytes (Ginhoux and Jung, 2014). Macrophages in atherosclerotic lesions by ingesting the accumulated lipoproteins, can be converted into "foam cells" which are filled with lipid droplets. Notably, these cholesterol-engorged foam cells that accumulate in atherosclerotic plaques appear to have decreased emigrational capacity. Due to this, inflammation in atherosclerotic plaque cannot be easily resolved, and further 
progress to more complex atherosclerotic plaque (Randolph, 2014). In these advanced plaques, macrophages further play role as a major contributor by maintaining the local inflammatory response through their secretion of pro-inflammatory mediators (chemokines, cytokines), and by producing reactive oxygen species. Inflammatory cytokines produced by macrophages induce the migration of more inflammatory cells to the lesion. Inflammatory macrophages even after their death play role in the formation of pro-thrombotic necrotic core by releasing lipid contents and tissue factor (Seimon and Tabas, 2009). Rupture of this necrotic core and the intravascular blood clot further contributes to myocardial infarction and stroke.

Although the association of macrophages with atherosclerosis has long been considered, the mechanism that regulates the formation of macrophage foam cells and their retention within the atheroma remains largely unknown (Randolph, 2014). Researchers are only beginning to elucidate the underlying mechanisms that control foam cell formation and retention within the plaque (Libby et al., 2014; Moore et al., 2013). In this review, we will discuss about the central roles of macrophages in the initiation, progression and resolution of atherosclerotic inflammation, by focusing on how the migration, recruitment, and death of macrophages alter the fate of the plaque.

\section{Recruitment of circulating monocytes in atherosclerosis}

During the past decade, the fact has become clear that hypercholesterolemia, an important risk factor for atherosclerosis, induces expansions of myeloid cells such as monocytes and neutrophils (Qiao et al., 1997). By studying different animal models, e.g. mice, swine and rabbits a significant correlation between augmented number of circulating monocytes and hyperlipidemia has been identified (Gu et al., 1998; Boring et al., 1998). In apolipoprotein E-deficient (Apoe ${ }^{-/}$) animals, circulating proinflammatory monocytes are found $\sim 50 \%$ higher than in the control animals (Swirski et al., 2007). Hence, mechanisms controlling monocyte recruitment is crucial to understand the contribution of monocyte in atherosclerosis.

Monocytes are bone marrow-derived progenitor cells which do not proliferate in the blood (Geissmann et al., 2003). During the process of atherosclerosis, endothelial injury leads to the migration of circulating monocytes to the intima and subintima (Cros et al., 2010). The rolling of monocytes to the sites of injury usually facilitates by the adhesion proteins of selectin family: P- and E-selectin (Blankenberg et al., 2003; Steffens and Mach, 2004). P-selectin is expressed on the luminal surface of endothelium and interacts with monocyte P-selectin glycoprotein ligand-1 (PSGL-1) (Blankenberg et al., 2003; Quehenberger, 2005). Firm adhesion of monocytes to the luminal surface of activated endothelium further supports by the binding of integrins with vascular cell adhesion molecule-1 (VCAM-1) and intercellular adhesion molecule-1 (ICAM-1) (Natarajan and Cai, 2005). Animal model studies have found that genetic deficiencies of VCAM-1 and ICAM-1 are associated with significantly delayed atherosclerosis (Takahashi et al., 2002).

In mice by tracing radiolabeled monocytes at the lesion site it has been recognized that monocytes can differentiate into macrophages (Ginhoux and Jung, 2014). The differentiation of monocytes into macrophages is triggered mainly by two growth factors: granulocyte/macrophage colony stimulating factor (GM-CSF) and macrophage colony-stimulating factor (M-SCF) (Nicola and Metcalf, 1986). Different human and animal model experiments have revealed that M-CSF overexpression play a key role to induce various functions of macrophage in the process of atherogenesis (Qiao et al., 1997). Moreover, delayed atheroma formation also has been documented in mice lacking the expression of M-CSF (Rajavashisth et al., 1998). The event of monocytes differentiation into macrophages also accompanied by a number of morphological and structural changes, such as increase of organelles numbers, altered sensitivity to signalling molecules, and enhanced expression of surface receptors including SR-AI and II, CD (cluster of differentiation) 36, MARCO, SR-PSOX, and CD68 (Kume and Kita, 2001; Minami et al., 2001). Differentiating monocytes show increased activity of lysosomal enzyme, which help them to prepare for the phagocytosis of lipid components (Novoselov et al, 2015). Macrophage after differentiating from monocytes further actively contributes to atherosclerotic lesion regression and tissue remodeling. In summary, monocyte recruitment to the arterial wall initiates the inflammation process (Nathan and Ding, 2010).

\section{Role of different macrophages in atherosclerosis}

Differentiated macrophages play a central role in atherosclerosis by scavenging lipoprotein particles and ultimately becoming foam cells (Lusis, 2000). Diverse emerging concepts on heterogeneity of macrophages indicate alternative roles of different macrophages in the progression and regression of atherosclerosis (Waldo $e t$ al., 2008; Bouhlel et al., 2007).

Existence of two types of macrophage phenotype is widely known- one is classically activated or M1 phenotype, and the other is alternatively activated or M2 phenotype (Johnson et al., 2009). Differentiation of 
M1 macrophages is occurred in response to toll-like receptor (TLR), interferon- $\gamma$ signaling; which is influenced by the availability of pathogen-associated molecular complexes (PAMPs), lipolysaccharides and lipoproteins. Proinflammatory factors, such as tumor necrosis factor- (TNF-) $\alpha$, interleukin-1 $\beta$ (IL-1 $\beta$ ), IL-12, and IL-23, and chemokines CXCL9, CXCL10, and CXCL11 are discharged by this type of macrophages. M1 macrophages produce high levels of reactive oxygen species (ROS) and nitric oxide (NO) that also contribute to the inflammation process (Chistiakov et al., 2015). Again various in vitro data show that M1 macrophages not only can trigger apoptosis of smooth muscle cells (SMCs) through activated Fas apoptotic pathway and release proapoptotic TNF $\alpha$ and nitric oxide, but also can decrease collagen synthesis in intimal SMCs through the secretion of macrophage-derived matrix metalloproteinases (MMPs) (Fodok et al., 1998).

Anti-inflammatory M2 macrophages are induced by Th2-type cytokines IL4 and IL-13 and secrete antiinflammatory factors, such as IL-1 receptor agonist and IL-10 (Martinez et al., 2009). Various studies have disclosed inaccuracy of bipolar M1/M2 classification to interpret macrophage diversity and so further division of M2 type into other subgroups was proposed by some authors based on the stimulation and protein expression pattern (Murray et al., 2014; Martinez et al., 2009). M2a, M2c play anti-inflammatory role while M2b play both anti and pro-inflammatory function and M2d is found to be involved in tumor progression (Ferrante et al., 2013).

Apart from M1 and M2 phenotypes, Mox phenotypes can also be evolved when exposed to oxidized phospholipids which are stimulated by functional transcription factor NFE2L2. Some pro-inflammatory markers, such as IL- $1 \beta$ and cyclooxygenase 2 are being secreted by Mox. In atherosclerotic lesions of mice, Mox macrophages comprise approximately $30 \%$ of the total number of macrophages. However, presence of Mox macrophages in human atherosclerotic lesions are yet to be determined (Kadl et al., 2010).

\section{Role of cytokines secreted by macrophages in atherosclerosis}

Macrophages are the first local inflammatory cells arrive at the atherosclerotic lesions (Gerrity et al., 1979). Macrophages secrete inflammatory cytokines that stimulate the generation of protease, endothelial adhesion molecules, and other mediators, which may enter into the systemic circulation in soluble forms (Galkina and Ley, 2009). Depending on their effects on the formation and progression of the atherosclerotic plaque, cytokines can be classified broadly as pro-atherogenic or anti-atherogenic. Pro-atherogenic cytokines (TNF- $\alpha$, IL-1, and IL-6) are secreted by macrophages, lymphocytes, NK-Cell and vascular smooth muscle cell and play greater role in atherogenesis by their overexpression (Tedgui and Mallat, 2006). In contrast, anti-atherogenic cytokines act in a protective manner against the atherosclerotic plaque formation such as transforming growth factor- $\beta$ (TGF- $\beta$ ) (Rajasingh et al., 2006).

TNF- $\alpha$ as a pro-atherogenic cytokines controls several critical cell functions such as cell proliferation, growth, survival, differentiation, and apoptosis. TNF- $\alpha$ binds with TNF receptor (TNFR) (Flynn et al., 1995) and acts as a "master regulator" of other pro-inflammatory cytokine production (Maini et al., 1995). Witsell and Schook (Witsell and Schook, 1992) illustrated that TNF- $\alpha$ also has macrophage differentiation capabilities. The expressions of TNF- $\alpha$ gene transcript occur mainly during the differentiation of bone marrow-derived macrophages.

IL-1 stimulation initiates leukocyte adhesion to endothelial cells for macrophage transmigration and also contributes to slowly progressing inflammatory processes which take place in atherosclerosis (Dinarello, 2011). Previous studies have documented that IL-1 expression induces inflammation in atherosclerosis, whereas Interleukin 1 receptor antagonist (IL-1RA) deficiency significantly promotes the disease (Elhage et al., 1998). The administration of recombinant IL-1RA into Apoe ${ }^{-/}$mice (Razin, 1984), or IL-1RA overexpression in Ld1 $\mathrm{r}$ ${ }^{1-}$ (Elhage et al., 1998) or Apoe ${ }^{-/}$mice, notably suppress plaque burden. On the contrary, IL-1RA knockout C57BL/6J mice fed a high fat diet tended to accumulate foam cells in the aortic wall and, thus, had accelerated disease. Although the circulating levels of IL-1, even in severe inflammatory disease, are unpredictable, with further research anti-IL-1 antibodies will likely be proposed as potential therapy for atherosclerotic patients in the future (Dinarello, 2011).

IL-12 is a heterodimeric cytokine that is produced mainly by plaque macrophages and have influence in $\mathrm{T}$ helper type 1 (Th1) differentiation, NK cells and T cells proliferation. Administration of IL-12 showed increased lesion size in Apoe ${ }^{-/-}$recipients. On the other hand, IL-12 p40-deficient I112b ${ }^{-/-}$Apoe $^{-/-}$mice have a $52 \%$ reduction of the plaque area at 30 weeks (Davenport and Tipping, 2003). Similarly, as IL-1 and IL-12, IL-8 is a pro-inflammatory cytokine which is secreted by macrophages and after administrating IL-8 antibody in Apoe $^{-/-}$mice it exacerbates the development of atherosclerotic lesions.

CD40 is produced by macrophages, ECs, and SMCs from atherosclerosis-prone vessels and has led to the hypothesis that the CD40L/CD40 dyad may contribute to atherosclerosis (Galkina and Ley, 2009). CD40L and 
CD40 were identified in human atherosclerotic lesions at very early developmental stages and their interaction plays a role in thrombosis, but it also contributes to the modulation of immune response to plaques formation. Treatment with antibodies against $\mathrm{CD} 40 \mathrm{~L}$ decreases atherosclerosis in $\mathrm{Ld}^{-\mathrm{r}^{--}}$mice, with a concomitant suppression of macrophages and T cells (Mach et al., 1998). Further experiments using Cd401g ${ }^{-/-}$Apoe $^{-/-}$mice have depicted a pro-atherogenic activity for $\mathrm{CD} 40 \mathrm{~L}$ in advanced atherosclerosis by developing lipid core formation and plaque destabilization (Zirlik et al., 2007). Although CD40 found critical for the development of advanced lesions in animal experiments, subclinical study conducted on a patient group of atherosclerosis did not find similar result (de Lemos et al., 2005). To successfully target cytokines and cytokine receptors as future therapeutic options, further studies are required to better understand the association of cytokines in atherosclerotic plaque formation.

\section{Role of chemokines in monocyte and macrophage migration to the atherosclerotic site}

Monocyte and macrophage homeostasis are tightly controlled by the chemoattractants including Complement component 5a (C5a) and (C-C motif) ligand 2 (CCL2) (Figure 1). C5a is one of the most potent inflammatory chemoattractants which enhances monocyte/macrophage recruitment to the site of inflammation (Distelmaier $e t$ al., 2009). It has the capability to induce the expression and release of abundant cytokines and chemokines including CCL2, CCL5 and CXCL1 (Buono et al., 2002).

CXCL1 plays major role in classical monocytosis and induced by high fat diet (Broxmeyer et al., 1996). CCL5 (RANTES) have activity in the recruitment of classical monocytes. With the acceleration of atherosclerosis in response to myocardial infarction, it is interesting to note that a recent clinical study evidenced an interaction between plasma CCL5 levels and progression of atherosclerosis after acute coronary syndrome (Blanchet $e t$ al., 2014).

CCL2, also defined as MCP-1, is also thought to be a major chemokine which has role in monocyte and macrophage recruitment during atherosclerosis development. Mouse model lacking CCL2 or its receptor develop smaller atherosclerotic plaques formation in comparison to the control. CCL2 induces monocyte mobilization from the sites of production. The lesion phenotype observed in CCR2-deficient mice showed low circulating monocyte numbers rather than the control mice (Buono et al., 2002).

Receptors of C5a are expressed by various cell types, such as macrophages, SMCs, mast cells, and endothelial cells. Inhibition of C5aR signaling decreased atherosclerosis and neointima formation in apoe $e^{-/-}$mice (Manthey et al., 2011). Due to its significant impacts on a neuromas cell types within the atherosclerotic plaque, C5a has identified as one of the key components in plaque destabilization and acute plaque rapture. Study also has been illustrated that enhanced levels of C5a are associated with increased cardiovascular risk in patients with advanced atherosclerosis (Speidl et al., 2005).

\section{Foam cell formation, macrophage death and its clearance by efferocytosis}

As discussed earlier, macrophage foam cells formation in the intima is a major hallmark of early stages of atherosclerotic plaque development. The significant cause of foam cells generation is the uncontrolled uptake of oxidized low-density lipoproteins (LDL) (Hutchins and Heinecke, 2015). Phagocytosis involved in the lipid internalization that is transported to late endosome/lysosomes, where lysosomal acid lipase (LAL) digests cholesteryl esters releasing free cholesteryl. Free cholesterol in turn is finally processed by acetyl-CoA acetyltransferase (ACAT1), an enzyme that help to transforms cholesteryl to cholesteryl esters (Chistiakov et al., 2016). The endoplasmic reticulum serves as a depot for controlling newly synthesized cholesteryl ester hydrolase (NCEH). In atherosclerosis, pro-inflammatory cytokines influence upregulates the expression of scavenger receptors, especially LOX-1, and downregulate the expression of cholesterol transporters. ACAT1 is also enhanced, while NCEH expression is reduced. This results in deposition of free and esterified cholesterol in macrophages and ultimately generation of foam cells. Furthermore, other cell types, such as endothelial (ECs) and vascular smooth muscle cells (VSMCs), can also resulting in foam cells (Chistiakov et al., 2017).

Formation of lipid rich necrotic cores within vulnerable plaques and macrophage death act as key factors in the promotion of advanced plaque necrosis. On a cellular level, "post-apoptotic," or "secondary," necrosis refers to a type of cell perturbation in where membranes become leaky and organelles swell, ultimately results in cellular death. In vivo experiment indicates that cell necrosis results when apoptotic cells in which membranes are primarily intact, but organelles are condensed, are not rapidly ingested by neighboring phagocytes. "Necrotic core" is often considered in the literature as "lipid core", because the dying macrophages are filled with lipid and mostly cholesterol, which ultimately becomes incorporated as extracellular lipid into the areas of plaque necrosis (Chistiakov et al., 2017; Tabas, 2005). 
Efferocytosis (Greek for carrying the corpse to the grave) is the removal of apoptotic cells by phagocytosis (Ortega-Gómez et al., 2013). Several stages can be distinguished in efferocytosis such as attraction of phagocytes, recognition, engulfment, and post-engulfment responses. Apoptotic cells (ACs) secrete soluble attraction or "find me" signals to in order to attract phagocytes and to initiate their own elimination. One such signal is the lipid lysophosphatidylcholine, which is mainly produced by caspase-3-activated phospholipase A2 and prompt clearance of the death cells (Lauber et al., 2003; Mueller et al., 2007). Sphingosine-1-phosphate (S1P) is formed by following caspase activation (Gude et al., 2008). Production of sphingosine kinase is upregulated in a caspase-dependent manner, which leads to extreme expression of S1P (Gude et al., 2008). Previous studies indicated that S1P is a great attractant for monocytes as well as macrophages (Gu et al., 2011). In fact, S1P has significant role on the regulation of epithelial cell extrusion during apoptosis (Gu et al., 2015). A more complete list of "come and get me" factors which can be found in the recent review by Peter et al. (2010). The leaky cell membrane of necrotic or late ACs liberates additional proteins, so-called alarmins, which propagate a danger signal and alert the immune system. Consequently, ACs gives electric signals which attract phagocytes, as has been mentioned for endothelial cells (Segundo et al., no date). As previous reviewed, (Thorp, 2010) in atherosclerosis an overload of these "eat me" signals can be found. In addition to "find me" signals, ACs will also express "eat me" signals, i.e., molecules that are upregulated or translocated to the surface to facilitate interactions with phagocytes. In fact, efferocytosis will largely depend on the quantity of positive ("eat me") and negative ("don't eat me") signals. In the end, the "phagocytic synapse" tightly interconnects the phagocyte with the dying cell (zipper like interaction). Therefore, ingestion by the phagocyte, the internalized AC will be processed and degraded through a series of fusions with endosomes and lysosomes (Soehnlein et al., 2013). Finally, as mentioned above, the impact of apoptosis is beneficial in early stages of atherosclerosis, whereas in advanced lesions, it results in plaque instability and thrombogenicity (Broxmeyer et al., 1991). It is assumed that less efficient scavenging of ACs and subsequent secondary necrosis play a significant function in unstable atherosclerotic lesions.

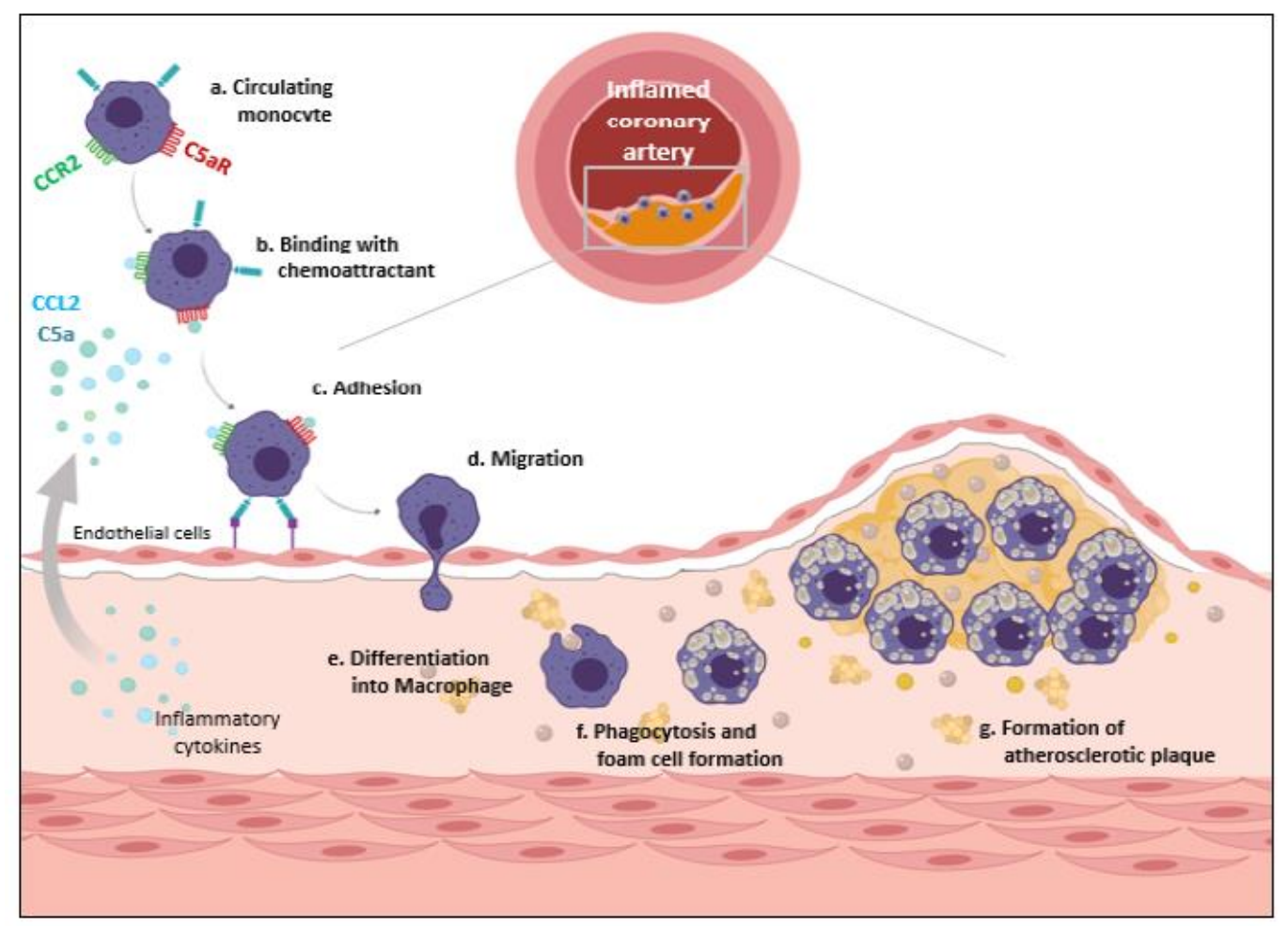

Figure 1. Monocyte trafficking from the circulation to the atherosclerotic plaque. (a) The accumulation of monocytes from the circulation to the atherosclerotic lesion is guided by inflammatory chemoattractants (such as CCL2 and C5a). (b) The engagement of chemoattractant with its receptor on monocyte surface facilitates its chemotaxis to the atherosclerotic endothelium. (c, d) By interacting with atherosclerotic endothelium, inflammatory monocytes then migrate into the atherosclerotic lesion. (e) Once they are recruited to the lesion site monocytes further contribute to inflammation by differentiating into macrophages. (f) These macrophages become foam cells by ingesting cholesterol. (g) These foam cells can form atherosclerotic plaque that may lead to atherosclerosis. 


\section{Conclusions}

Atherosclerosis is a chronic inflammatory disease arising from an imbalance in lipid metabolism and a maladaptive immune response driven by the accumulation of cholesterol-laden macrophages in the artery wall. Concepts on how macrophages and their related cytokines and other factors affect different stages of atherosclerosis is emphasized in this review because highlighting the association of macrophage with atherosclerosis is critical for understanding the prognosis, diagnosis, and treatment of this disease. Macrophages are a vital and versatile lineage having numerous associated effector functions that implicate them in several features of atherosclerosis including lipid phagocytosis, plaque repair, plaque rupture, and the autoimmune phenomena associated with atherosclerosis. After being recruited by circulated monocytes, they take part in LDL uptake and lipid accumulation in the arterial wall becoming foam cell. Macrophage population is heterogenous and several subtypes differ in their function and gene expression profiles. This review provides a brief discussion on the involvement of macrophages in atherosclerosis mainly based on animal model studies which may not completely reflect the process in human. To discover more effective and potential therapeutic options for atherosclerosis, in future more human genetic studies along with mechanism based clinical trials should be performed.

\section{Conflict of interest}

None to declare.

\section{References}

Back M and GK Hansson, 2015. Anti-inflammatory therapies for atherosclerosis. Nat. Rev. Cardiol., 12: 199211.

Broxmeyer HE, S Cooper, G Cacalano, NL Hague, E Bailish and MW Moore, 1996. Involvement of Interleukin (IL) 8 receptor in negative regulation of myeloid progenitor cells in vivo: evidence from mice lacking the murine IL-8 receptor homologue. J. Exp. Med., 184: 1825-1832.

Blanchet X, K Cesarek, J Brandt, H Herwald, D Teupser, H Kuechenhoff, E Karshovska, SF Mause, W Siess, H Wasmuth and O Soehnlein, 2014. Inflammatory role and prognostic value of platelet chemokines in acute coronary syndrome. Thromb Haemost., 112: 1277-1287.

Blankenberg S, S Barbaux and L Tiret, 2003. Adhesion molecules and atherosclerosis. Atherosclerosis, 170: 191-203.

Boring L, J Gosling and M Cleary, 1998. Decreased lesion formation in CCR2 ${ }^{-/-}$mice reveals a role for chemokines in the initiation of atherosclerosis. Nature, 394: 894-897.

Bouhlel MA, B Derudas, E Rigamonti, R Dièvart, J Brozek, S Haulon, C Zawadzki, B Jude, G Torpier, N Marx and B Staels, 2007. PPAR $\gamma$ activation primes human monocytes into alternative M2 macrophages with antiinflammatory properties. Cell Metab., 6: 137-143.

Broxmeyer HE, B Sherry, S Cooper, FW Ruscetti, DE Williams, P Arosio, BS Kwon and A Cerami, 1991. Macrophage inflammatory protein (MIP)-1 beta abrogates the capacity of MIP-1 alpha to suppress myeloid progenitor cell growth. J. Immunol., 147: 2586-2594.

Broxmeyer HE, S Cooper, G Cacalano, NL Hague, E Bailish and MW Moore, 1996. Involvement of Interleukin (IL) 8 receptor in negative regulation of myeloid progenitor cells in vivo: evidence from mice lacking the murine IL-8 receptor homologue. J. Exp. Med., 184: 1825-1832.

Buono C, CE Come, JL Witztum, GF Maguire, PW Connelly, M Carroll and AH Lichtman, 2002. Influence of C3 deficiency on atherosclerosis. Circ. Res., 105: 3025-3031.

Chistiakov DA, YV Bobryshev, NG Nikiforov, NV Elizova, IA Sobenin and AN Orekhov, 2015. Macrophage phenotypic plasticity in atherosclerosis: the associated features and the peculiarities of the expression of inflammatory genes. Int. J. Cardiol., 184: 436-445.

Chistiakov DA, AA Melnichenko, VA Myasoedova, AV Grechko and AN Orekhov, 2017. Mechanisms of foam cell formation in atherosclerosis. J. Mol. Med., 95: 1153-1165.

Chistiakov DA, YV Bobryshev and AN Orekhov, 2016. Macrophage-mediated cholesterol handling in atherosclerosis. J. Cell Mol. Med., 20: 17-28.

Cros J, N Cagnard and K Woollard, 2010. Human CD14 ${ }^{\mathrm{dim}}$ monocytes patrol and sense nucleic acids and viruses via TLR7 and TLR8 receptors. Immunity, 33: 375-386.

Dahlöf B. 2010. Cardiovascular disease risk factors: epidemiology and risk assessment. Am. J. Cardiol., 105: 3A-9A.

Davenport P and PG Tipping, 2003. The role of interleukin-4 and interleukin-12 in the progression of atherosclerosis in apolipoprotein E-deficient mice. Am. J. Pathol., 163: 1117-1125. 
De Lemos JA, A Zirlik, U Schönbeck, N Varo, SA Murphy, A Khera, DK McGuire, G Stanek, HS Lo, R Nuzzo and DA Morrow, 2005. Associations between soluble CD40 ligand, atherosclerosis risk factors, and subclinical atherosclerosis: results from the Dallas Heart Study. Arterioscler. Thromb. Vasc. Biol., 25: 21922196.

Dinarello CA, 2011. A clinical perspective of IL-1 $\beta$ as the gatekeeper of inflammation. Eur. J. Immunol., 41: 1203-1217.

Distelmaier K, C Adlbrecht, J Jakowitsch, S Winkler, D Dunkler, C Gerner, O Wagner, IM Lang and M Kubicek, 2009. Local complement activation triggers neutrophil recruitment to the site of thrombus formation in acute myocardial infarction. Thromb. Haemost., 102: 564-572.

Elhage R, A Maret, MT Pieraggi, JC Thiers, JF Arnal and F Bayard, 1998. Differential effects of interleukin-1 receptor antagonist and tumor necrosis factor binding protein on fatty-streak formation in apolipoprotein Edeficient mice. Circulation, 97: 242-244.

Fadok VA, DL Bratton, A Konowal, PW Freed, JY Westcott and PM Henson, 1998. Macrophages that have ingested apoptotic cells in vitro inhibit proinflammatory cytokine production through autocrine/paracrine mechanisms involving TGF-beta, PGE2, and PAF. J. Clin. Invest., 101: 890-898.

Ferrante CJ, G Pinhal-Enfield, G Elson, BN Cronstein, G Hasko, S Outram and SJ Leibovich, 2013. The adenosine-dependent angiogenic switch of macrophages to an M2-like phenotype is independent of interleukin-4 receptor alpha (IL-4R $\alpha$ ) signaling. Inflammation, 36: 921-931.

Flynn JL, MM Goldstein, J Chan, KJ Triebold, K Pfeffer, CJ Lowenstein, R Schrelber, TW Mak and BR Bloom, 1995. Tumor necrosis factor- $\alpha$ is required in the protective immune response against Mycobacterium tuberculosis in mice. Immunity, 2: 561-572.

Galkina E and K Ley, 2009. Immune and inflammatory mechanisms of atherosclerosis. Annu. Rev. Immunol., 27.

Geissmann F, S Jung, DR Littman, 2003. Blood monocytes consist of two principal subsets with distinct migratory properties. Immunity, 19: 71-82.

Gerrity RG, HK Naito, M Richardson and CJ Schwartz, 1979. Dietary induced atherogenesis in swine: morphology of the intima in prelesion stages. Am. J. Pathol., 95: 775.

Ginhoux F and S Jung, 2014. Monocytes and macrophages: developmental pathways and tissue homeostasis. Nat. Rev. Immunol., 14: 392-404.

Gronholdt ML, S Dalager-Pedersen and E Falk, 1998. Coronary atherosclerosis: determinants of plaque rupture Eur. J. Heart Suppl., C. 4: 24-29.

Gu Y, J Shea, G Slattum, MA Firpo, M Alexander and SJ Mulvihill, 2015. Defective apical extrusion signaling contributes to aggressive tumor hallmarks. Life, 4: 04069.

Gu Y, T Forostyan, R Sabbadini and J Rosenblatt, 2011. Epithelial cell extrusion requires the sphingosine-1phosphate receptor 2 pathway. J. Cell Biol., 193: 667-676.

Gu L, Y Okada and S Clinton, 1998. Absence of monocyte chemoattractant protein-1 reduces atherosclerosis in low-density lipoprotein-deficient mice. Mol. Cell., 2: 275-281.

Gude DR, SE Alvarez, SW Paugh, P Mitra, J Yu and Griffiths R, 2008. Apoptosis induces expression of sphingosine kinase 1 to release sphingosine-1-phosphate as a "come-and-get-me" signal. FASEB J., 22: 2629-2638.

Hutchins PM and JW Heinecke, 2015. Cholesterol efflux capacity, macrophage reverse cholesterol transport, and cardioprotective HDL. Curr. Opin. Lipidol., 26: 388.

Johnson JL and AC Newby, 2009. Macrophage heterogeneity in atherosclerotic plaques. Curr. Opin. Lipidol., 20: 370.

Kadl A, AK Meher, PR Sharma, MY Lee, AC Doran, SR Johnstone, MR Elliott, F Gruber, J Han, W Chen and $\mathrm{T}$ Kensler, 2010. Identification of a novel macrophage phenotype that develops in response to atherogenic phospholipids via Nrf2. Circ. Res., 107: 737-746.

Krauss RM, 2010. Lipoprotein subfractions and cardiovascular disease risk. Cur. Opin. Lipidol., 21: 305-311.

Kume $\mathrm{N}$ and $\mathrm{T}$ Kita, 2001. Roles of lectin-like oxidized LDL receptor-1 and its soluble forms in atherogenesis. Curr. Opin. Lipidol., 12: 419-423.

Lauber K, E Bohn, SM Krober, YJ Xiao, SG Blumenthal and RK Lindemann, 2003. Apoptotic cells induce migration of phagocytes via caspase-3-mediated release of a lipid attraction signal. Cell, 113: 717-730.

Libby P, I Tabas, G Fredman and EA Fisher, 2014. Inflammation and its resolution as determinants of acute coronary syndromes. Circ. Res., 114: 1867-187.

Lloyd-Jones DM, 2010. Cardiovascular risk prediction: basic concepts, current status, and future directions. Circulation, 121: 1768-1777. 
Lusis AJ, 2012. Genetics of atherosclerosis. Trends. Genet., 28: 267-275.

Mach F, U Schönbeck, GK Sukhova, E Atkinson and P Libby, 1998. Reduction of atherosclerosis in mice by inhibition of CD40 signalling. Nature, 394: 200.

Maini RN, MJ Elliott, FM Brennan and M Feldmann, 1995. Beneficial effects of tumour necrosis factor-alpha (TNF- $\alpha$ ) blockade in rheumatoid arthritis (RA). J. Clin. Exp. Immunol., 101: 207-212.

Manthey HD, AC Thomas, IA Shiels, A Zernecke, TM Woodruff, B Rolfe and SM Taylor, 2011. Complement C5a inhibition reduces atherosclerosis in ApoE-/- mice. FASEB J., 25: 2447-2455.

Martinez FO, L Helming and S Gordon, 2009. Alternative activation of macrophages: an immunologic functional perspective. Annu. Rev. Immunol., 27: 451-483.

Minami M, N Kume, T Shimaoka, H Kataoka, K Hayashida, Y Akiyama, I Nagata, K Ando, M Nobuyoshi, M Hanyuu, M Komeda, S Yonehara and T Kita, 2001. Expression of SR-PSOX, a novel cell-surface scavenger receptor for phosphatidylserine and oxidized LDL in human atherosclerotic lesions. Arterioscler. Thromb. Vasc. Biol., 21: 1796-1800.

Mueller RB, A Sheriff, US Gaipl, S Wesselborg and K Lauber, 2007. Attraction of phagocytes by apoptotic cells is mediated by lysophosphatidylcholine. Autoimmunity, 40: 342-344.

Moore KJ, FJ Sheedy and EA Fisher, 2013. Macrophages in atherosclerosis: a dynamic balance. Nat. Rev. Immunol., 13:709-721.

Murray PJ, JE Allen, SK Biswas, EA Fisher, DW Gilroy, S Goerdt, S Gordon, JA Hamilton, LB Ivashkiv, T Lawrence and M Locati, 2014. Macrophage activation and polarization: nomenclature and experimental guidelines. Immunity, 41: 14-20.

Natarajan R and Q Cai, 2005. Monocyte retention in the pathology of atherosclerosis. Future Cardiol., 1: 331340.

Nathan C and A Ding, 2010. Nonresolving inflammation. Cell, 140: 871-882.

Nicola NA and D Metcalf, 1986. Specificity of action of colony-stimulating factors in the differentiation of granulocytes and macrophages. Ciba. Found. Symp., 118: 7-28.

Novoselov VV, MA Sazonova MA, EA Ivanova and AN Orekhov, 2015. Study of the activated macrophage transcriptome. Exp. Mol. Pathol., 99: 575-580.

Ortega-Gómez A, M Perretti and O Soehnlein, 2013. Resolution of inflammation: an integrated view. EMBO Mol. Med., 5: 661-674.

Peter C, S Wesselborg, M Herrmann and K Lauber, 2010. Dangerous attraction: phagocyte recruitment and danger signals of apoptotic and necrotic cells. Apoptosis, 15: 1007-1028.

Qiao JH, J Tripathi, NK Mishra, Y Cai, S Tripathi, XP Wang, S Imes, MC Fishbein, SK Clinton, P Libby, AJ Lusis and TB Rajavashisth. 1997. Role of macrophage colony-stimulating factor in atherosclerosis: studies of osteopetrotic mice. Am. J. Pathol., 150: 1687-1699.

Quehenberger O, 2005. Molecular mechanisms regulating monocyte recruitment in atherosclerosis. J. Lipid Res., 46: 1582-1590.

Rajasingh J, E Bord, C Luedemann, J Asai, H Hamada, T Thorne, G Qin, D Goukassian, Y Zhu, DW Losordo and R Kishore, 2006. IL-10-induced TNF-alpha mRNA destabilization is mediated via IL-10 suppression of p38 MAP kinase activation and inhibition of HuR expression. FASEB J., 20: 2112-2114.

Rajavashisth T, JH Qiao, S Tripathi, J Tripathi, N Mishra, M Hua, XP Wang, A Loussararian, S Clinton, P Libby and A Lusis. 1998. Heterozygous osteopetrotic (op) mutation reduces atherosclerosis in LDL receptordeficient mice. J. Clin. Invest., 101: 2702-271.

Randolph GJ. 2014. Mechanisms that regulate macrophage burden in atherosclerosis. Circ. Res., 114: 17571771.

Razin E and G Marx, 1984. Thrombin-induced degranulation of cultured bone marrow-derived mast cells. J. Immunol., 133: 3282-3285.

Segundo C, F Medina, C Rodríguez, R Martínez-Palencia, F Leyva-Cobián and JA Brieva, 1999. Surface molecule loss and bleb formation by human germinal center B cells undergoing apoptosis: role of apoptotic blebs in monocyte chemotaxis. Blood, 94: 1012-1020.

Seimon T and I Tabas, 2009. Mechanisms and consequences of macrophage apoptosis in atherosclerosis. J. Lipid Res., 50: 382-387.

Shah PK, 2002. Pathophysiology of coronary thrombosis: role of plaque rupture and plaque erosion Prog. Cardiovasc. Dis., 44: 357-368.

Soehnlein O, M Drechsler, Y Döring, D Lievens, H Hartwig and K Kemmerich, A Ortega-Gómez, M Mandl, S Vijayan, D Projahn and CD Garlichs, 2013. Distinct functions of chemokine receptor axes in the atherogenic mobilization and recruitment of classical monocytes. EMBO Mol. Med., 5: 471-481. 
Speidl WS, M Exner, J Amighi, SP Kastl, G Zorn, G Maurer, O Wagner, K Huber, E Minar, J Wojta, and M Schillinger, 2005. Complement component C5a predicts future cardiovascular events in patients with advanced atherosclerosis. Eur. Heart J., 26: 2294-2299.

Steffens S and F Mach, 2004. Inflammation and atherosclerosis. Herz., 29: 741-748.

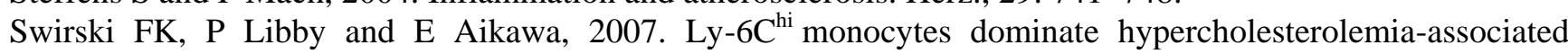
monocytosis and give rise to macrophages in atheromata. J. Clin. Invest., 117: 195-205.

Tabas I, 2005. Consequences and therapeutic implications of macrophage apoptosis in atherosclerosis: the importance of lesion stage and phagocytic efficiency. Arterioscl. Thromb. Vas., 25: 2255-2264.

Takahashi K, M Takeya and N Sakashita, 2002. Multifunctional roles of macrophages in the development and progression of atherosclerosis in humans and experimental animals. Med. Electron Microsc., 35: 179-203.

Tedgui A and Z Mallat, 2006. Cytokines in atherosclerosis: pathogenic and regulatory pathways. Physiol. Rev., 86: 515-581.

Thorp EB, 2010. Mechanisms of failed apoptotic cell clearance by phagocyte subsets in cardiovascular disease. Apoptosis, 15: 1124-1136.

Waldo SW, Y Li, C Buono, B Zhao, EM Billings, J Chang, and HS Kruth, 2008. Heterogeneity of human macrophages in culture and in atherosclerotic plaques. Am. J. Pathol., 172: 1112-1126.

Weber C, A Zernecke and P Libby, 2008. The multifaceted contributions of leukocyte subsets to atherosclerosis: lessons from mouse models. Nat. Rev. Immunol., 8: 802-815.

Witsell AL and LB Schook, 1992. Tumor necrosis factor alpha is an autocrine growth regulator during macrophage differentiation. Proc. Natl. Acad. Sci., 89: 4754-4758.

Zirlik A, C Maier, N Gerdes, L MacFarlane, J Soosairajah, U Bavendiek, I Ahrens, S Ernst, N Bassle, A Missiou and Z Patko 2007. CD40 ligand mediates inflammation independently of CD40 by interaction with Mac-1. Circulation, 115: 1571. 